\title{
The 17 MeV Anomaly in Beryllium Decays and U(1) Portal to Dark Matter
}

\author{
Chian-Shu Chen ${ }^{* a}$, Guey-Lin $\operatorname{Lin}^{b}$, Yen-Hsun $\operatorname{Lin}^{b}$ and Fanrong $\mathbf{X u}^{c}$ \\ ${ }^{a}$ Department of Physics, Tamkang University \\ New Taipei City 25137, Taiwan \\ ${ }^{b}$ Institute of Physics, National Chiao Tung University \\ Hsinchu 30010, Taiwan \\ ${ }^{c}$ Department of Physics, Jinan University \\ Guangzhou 510632, P.R. China \\ E-mail: chianshu@gmail.com, glin@cc.nctu.edu.tw, \\ chris.py99g@g2.nctu.edu.tw, fanrongxuejnu.edu.cn
}

\begin{abstract}
The experiment of Krasznahorkay et al observed the transition of a ${ }^{8} \mathrm{Be}$ excited state to its ground state and accompanied by an emission of $e^{+} e^{-}$pair with $17 \mathrm{MeV}$ invariant mass. This $6.8 \sigma$ anomaly can be fitted by a new light gauge boson. We consider the new particle as a $U(1)$ gauge boson, $Z^{\prime}$, which plays as a portal linking dark sector and visible sector. In particular, we study the new $U(1)$ gauge symmetry as a hidden or non-hidden group separately. The generic hidden $U(1)$ model, referred to as dark $Z$ model, is excluded by imposing various experimental constraints. On the other hand, a non-hidden $Z^{\prime}$ is allowed due to additional interactions between $Z^{\prime}$ and Standard Model fermions. We also study the implication of the dark matter direct search on such a scenario. We found the search for the DM-nucleon scattering cannot probe the parameter space that is allowed by ${ }^{8} \mathrm{Be}$-anomaly for the range of DM mass above $500 \mathrm{MeV}$. However, the DMelectron scattering for DM between 20 and $50 \mathrm{MeV}$ can test the underlying $U(1)$ portal model using the future $\mathrm{Si}$ and $\mathrm{Ge}$ detectors with $5 e^{-}$threshold charges.
\end{abstract}

The European Physical Society Conference on High Energy Physics

5-12 July, 2017

Venice

${ }^{*}$ Speaker. 


\section{Introduction}

The experiment of Krasznahorkay et al. studied the decays of a ${ }^{8} \mathrm{Be}$ excited state to its ground state and found a bump in both the opening angle and invariant mass distributions of $e^{+} e^{-}$pairs produced in the transitions [1]. This $6.8 \sigma$ deviation from the expectation can be fitted by the production of a new particle $\mathrm{X}$ of mass around $17 \mathrm{MeV}$ in the transition ${ }^{8} \mathrm{Be}^{*} \rightarrow{ }^{8} \mathrm{Be} \mathrm{X}$ and the subsequent decay of $\mathrm{X}$ into electron-positron pair. In this study, we take the new particle as a $Z^{\prime}$ gauge boson corresponding to a new $U(1)_{d}$ gauge symmetry. We do not specify the new quantum number at the moment. After inputting a series experimental constraints, we will get some knowledge of possibility about the new quantum number $d$.

\section{The generic hidden $U(1)$ model}

The simplest case will be the complete gauge $U(1)$ hidden symmetry. The generic Lagrangian can be written as

$$
\mathscr{L}_{\text {gauge }}=-\frac{1}{4} B_{\mu v} B^{\mu v}+\frac{1}{2} \frac{\varepsilon}{\cos \theta_{W}} Z_{\mu v}^{\prime} B^{\mu v}-\frac{1}{4} Z_{\mu v}^{\prime} Z^{\prime \mu v}
$$

where $\varepsilon$ characterizes the kinetic mixing. After spontaneous symmetry breaking (SSB) $W$ and $Z$ bosons get their masses while photon stays massless in SM. Generically in addition to kinetic mixing interaction as shown eq. (2.1), there is a mass mixing term between $Z^{\prime}$ and $Z$. The electromagnetic current and weak neutral current are $J_{\mathrm{em}, f}^{\mu}=Q_{f} \bar{f} \gamma^{\mu} f$ and $J_{\mathrm{NC}, f}^{\mu}=\left(T_{3 f}-2 Q_{f} s_{W}^{2}\right) \bar{f} \gamma^{\mu} f-$ $T_{3 f} \bar{f} \gamma^{\mu} \gamma_{5} f$ respectively, where $f$ stands for the fermions with corresponding electric charge $Q_{f}$, isospin $T_{3 f}= \pm \frac{1}{2}$. We take $M_{Z^{\prime}}=16.7 \pm 0.35$ (stat.) \pm 0.5 (sys.) $\mathrm{MeV}$ as the possible new light boson according to Ref. [1]. Therefore, the $Z^{\prime}$ considered in our framework can only decay into $e^{+} e^{-}$and $v \bar{v}$.

As pointed in Ref. [2] and constraints summarized in Ref. [3], a protophobic feature is suggested by the measurement of Krasznahorkay et al. after taking into account a series experimental constraints such as NA48/2 [4] and E141 experiments [5]. However, for the hidden $U(1)$ model the same constraints also apply to $\left(a_{e}, a_{v}\right)\left(e-Z^{\prime}\right.$ and $v-Z^{\prime}$ couplings respectively) in the generic hidden $U(1)$ model. Therefore, the constraints from short baseline accelerator and/or reactor neutrinoelectron scattering experiments must be taken into account. These constraints are so stringent that they are incompatible with the experimental constraints in the framework of generic hidden $U(1)$ model (see Fig. 1). In other words, a complete hidden $U(1)$ portal as the explanation for the $8^{B} e$ anomaly is excluded.

\section{Non-hidden $U(1)$ portal and DM-electron scattering process}

To accommodate the new light gauge boson indicated in ${ }^{8} \mathrm{Be}$ anomaly as well as $U(1)$ portal scenario, we are led to consider models with non-hidden $U(1)$ gauge symmetry and MeV-scale DM. Non-hidden $U(1)$ charge suggests a certain linear combination of SM quantum number and/or other hidden charge. Phenomenologically, such models will include a new set of direct gaugefermion couplings. There are various ways of model-building to impose such non-hidden $U(1)$ gauge symmetry motivated by ${ }^{8} \mathrm{Be}$ anomaly $[6,7]$. In this paper, we do not intend to study these 


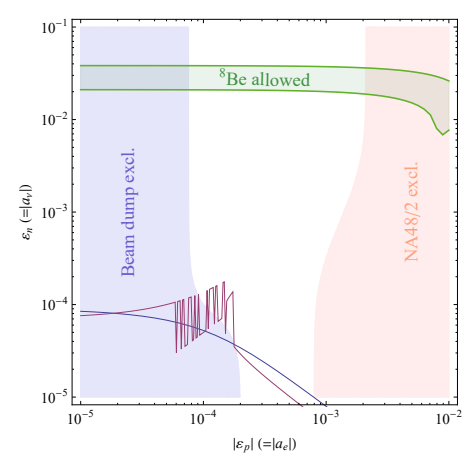

Figure 1: The allowed parameter space on $\left(\varepsilon_{p}, \varepsilon_{n}\right)$ or $\left(a_{e}, a_{v}\right)$ plane in generic hidden $U(1)$ model. E141 and NA48/2 exclusion regions are indicated. The green band is the allowed parameter space resulting from ${ }^{8} \mathrm{Be}$ anomaly with the error of $Z^{\prime}$ mass taken into account. The allowed narrow band is however incompatible with the TEXONO $v-e$ scattering experiment [8]. The purple line corresponds to the constraint of $\mid\left(a_{e}+\right.$ $\left.a_{v}\right) a_{v} \mid \lesssim 5 \times 10^{-9}$ and blue line corresponds to $\left|\left(a_{e}-a_{v}\right) a_{v}\right| \lesssim 8 \times 10^{-9}$. The upper regions of these two lines are excluded.

models in detail but rather assume that the couplings of $Z^{\prime}$ to various fermions are not correlated. In particular, we assume the severe constraint from $v-e$ scattering can be alleviated ${ }^{1}$.

\subsection{DM direct searches and the DM-nucleon cross section}

Even though the non-hidden $\mathrm{U}(1)$ models can accommodate both Be-anomaly and $v-e$ scattering experiments, it is worthy of looking at the impact of DM direct searches on these models. In the limit of zero momentum transfer $\left(q^{2}=0\right)$, the DM-nucleus scattering cross section is given by

$$
\sigma_{\chi \mathrm{A}}=\frac{16 \pi \alpha_{\mathrm{em}} \alpha_{d} \mu_{\chi A}^{2}}{M_{Z^{\prime}}^{4}}\left[\varepsilon_{p} Z+\varepsilon_{n}(A-Z)\right]^{2}
$$

where $\alpha_{d}=e_{d}^{2} / 4 \pi$ is the analogous fine structure constant for $U(1)_{d}$ gauge interaction, $\mu_{\chi A}=$ $m_{\chi} m_{A} /\left(m_{\chi}+m_{A}\right)$ is the DM-nucleus reduced mass, $Z$ and $A$ are proton number and mass number respectively. In this case, DM-neutron coupling $\varepsilon_{n}$ gives the main contribution to $\sigma_{\chi A}$ due to the suppression of $\varepsilon_{p}$ compared to $\varepsilon_{n}$. The inclusion of cosmic microwave background constraint, DM relic abundance, DM self-interaction and big bang nucleosynthesis etc. is considered in our original paper [9]. We refer readers to this reference for details.

We plot in Fig. 2(right) the theoretical predictions of $\sigma_{\chi n}$ for DM mass between 0.5-10 GeV. The DM-neutron cross section is around the $10^{-26} \mathrm{~cm}^{2}$ for $m_{\chi}=0.5 \mathrm{GeV}$. Although the direct searches have set strong bounds on $\sigma_{\chi n}$, we cannot naively apply such bounds. It is because the DM cannot reach the underground detectors for $\sigma_{\chi n} \sim 10^{-26} \mathrm{~cm}^{2}{ }^{2}$ This can be understood by considering a underground laboratory with $1000 \mathrm{~m}$ of standard rock (with $Z=11, A=22$ and the density $\rho=2.65 \mathrm{~g} / \mathrm{cm}^{3}$ ) as the overburden. For $m_{\chi}=1 \mathrm{GeV}$, the DM with $\sigma_{\chi_{n}}$ higher than $5.4 \times 10^{-30} \mathrm{~cm}^{2}$ will not be able to reach the detector since its attenuation length will be shorter

\footnotetext{
${ }^{1}$ One simple example is the $U(1)_{B}$ model with $B$ the baryon number. In such a model the neutrino- $Z^{\prime}$ coupling vanishes, thus the TEXONO bounds can be evaded. An anomaly free $U(1)_{B}$ model is proposed in Ref. [7]

${ }^{2}$ We thank the Referee for pointing out this to us.
} 

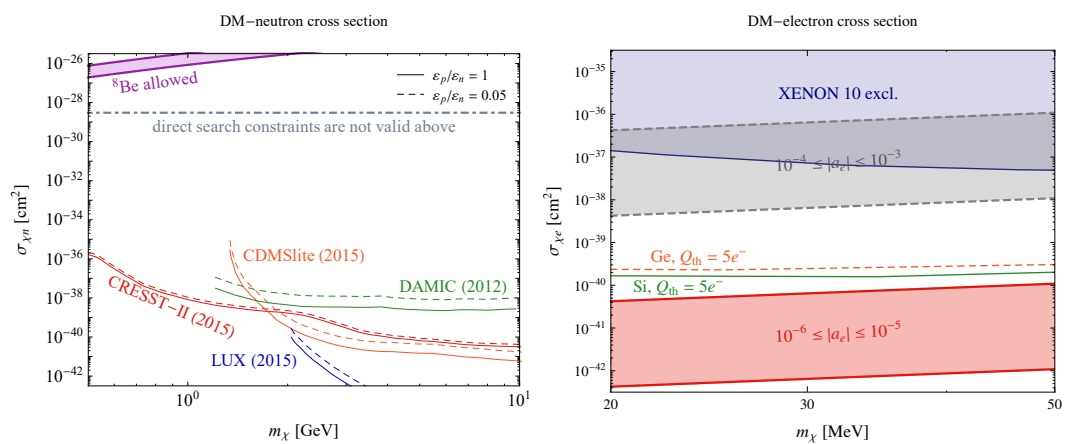

Figure 2: (left)The theoretical predictions of $\sigma_{\chi^{n}}$ from the allowed range of $\left(\varepsilon_{p}, \varepsilon_{n}\right)$ parameter space is shown. The purple shaded regions are the predicted DM-neutron cross section. The exclusion lines by CRESST-II(2015) [10], DAMIC(2012) [11], CDMSlite(2015) [12], and LUX(2015) [13] are presented for $\varepsilon_{p} / \varepsilon_{n}=1$ and $\varepsilon_{p} / \varepsilon_{n}=0.05$, respectively, where the latter ratio is the protophobic scenario favored by ${ }^{8} \mathrm{Be}$ experiment. $\sigma_{\chi n}$ is predected to be around $10^{-26} \sim 10^{-27} \mathrm{~cm}^{2}$. The attenuation length of DM with these cross section will not penetrate $1000 \mathrm{~m}$ rock to reach the detectors. We take the $1000 \mathrm{~m}$ depth as the benchmark, and draw the gray dashed-dotted line to represent this attenuation effect. The parameter space above this line will not be probed by the DM direct searches. Therefore, DM direct searches will not probe the parameter space allowed by Be-anomaly. (right)The red shaded regions are the theoretical predictions of $\sigma_{\chi e}$ with $\left|a_{e}\right|$ lying in the range of $10^{-6}-10^{-5}$ for $20 \mathrm{MeV}<m_{\chi}<50 \mathrm{MeV}$. The gray shaded region are the predictions with $\left|a_{e}\right|$ lying in the range of $10^{-4}-10^{-3}$. XENON 10 [14] excluded the parameter space is represented by purple shaded region. The projected sensitivities of $\mathrm{Si}$ and Ge detectors with threshold charges of $5 e^{-}$are represented by the green solid line and the orange dashed line, respectively.

than $1000 \mathrm{~m}$. On the other hand, a cross section lower than $5.4 \times 10^{-30} \mathrm{~cm}^{2}$ will be subject to the direct search constraint. Here attenuation length is defined as $\Lambda=1 /(n \sigma \eta)$ where $n$ is the number density of target nuclei and $\eta$ is the inelasticity of DM-rock collision, i.e., the fraction of initial DM kinetic energy transferred to the target nucleus. On average we have $\eta=m_{\chi} / m_{A}$. For a fixed standard rock overburden, the critical DM-neutron cross section, denoted as $\sigma_{\chi n}^{c}$, below which DM can reach to the detector is proportional to $m_{A} / m_{\chi} \cdot m_{n}^{2} /\left(m_{\chi}+m_{n}\right)^{2}$. Clearly $\sigma_{\chi n}^{c}$ increases as $m_{\chi}$ decreases.

\subsection{DM-electron scattering process}

We note that the conventional DM direct search looks for the nuclear recoils. However, the nuclear recoil energy, $E_{\text {recoil }}=\left(m_{\chi} v\right)^{2} /\left(2 m_{A}\right) \approx\left(m_{\chi} / 100 \mathrm{MeV}\right)^{2}\left(m_{A} / 10 \mathrm{GeV}\right)^{-1} \mathrm{eV}$, is sub-eV for the $\mathrm{MeV}$-scale DM and is far below the threshold energies in current experiments. The DM-electron cross section can be written as $\sigma_{\chi e}=16 \pi \alpha_{\mathrm{em}} \alpha_{d} a_{e}^{2} \frac{\mu_{\chi e}^{2}}{M_{Z^{\prime}}^{4}}, \mu_{\chi e}$ is the DM-electron reduced mass. We show the theoretical predictions to $\sigma_{\chi e}$ and XENON 10 exclusion region in Fig. 2(right). The purple shaded area is excluded by XENON 10 based on the DM-electron scattering and the capability of charge threshold $Q_{\mathrm{th}}=10 e^{-}$[14]. The projected sensitivities for Silicon and Germanium targets with improved $Q_{\mathrm{th}}=5 e^{-}$are presented as green solid line and orange dashed line, respectively [15]. The red shaded region is the theoretical predictions of $\sigma_{\chi e}$ corresponding to the range of $10^{-6}<$ $\left|a_{e}\right|<10^{-5}$. Our calculation on $\sigma_{\chi e}$ shows that the MeV DM under $Z^{\prime}$ model can be tested by future experiments. 


\section{Summary}

Motivated by the possible existence of a new light boson from the experiment of Krasznahorkay et al. [1], we investigate the $Z^{\prime}$-portal models. The reactor neutrino-electron scattering sets a severe constraint which excludes the parameter space of generic hidden $U(1)$ model. Using the current DM direct search data, we have shown that the non-hidden $U(1)$ models predict $\sigma_{\chi^{n}}$ to be larger than $10^{-27} \mathrm{~cm}^{2}$ for $m_{\chi} \geqslant 500 \mathrm{MeV}$. Such DM cannot reach the detector located beneath 1000 $\mathrm{m}$ of rock and cannot be probed by the DM-nucleon scattering approach. To probe DM lighter than $50 \mathrm{MeV}$ in non-hidden $U(1)$ models, we propose direct searches based upon DM-electron scatterings. The sensitivities of these detectors to the couplings strength $\left|a_{e}\right|$ can be determined accordingly.

\section{References}

[1] A. J. Krasznahorkay et al., Phys. Rev. Lett. 116, no. 4, 042501 (2016) doi:10.1103/PhysRevLett.116.042501 [arXiv:1504.01527 [nucl-ex]].

[2] J. L. Feng, B. Fornal, I. Galon, S. Gardner, J. Smolinsky, T. M. P. Tait and P. Tanedo, Phys. Rev. Lett. 117, no. 7, 071803 (2016) [arXiv:1604.07411 [hep-ph]].

[3] J. Alexander et al., arXiv:1608.08632 [hep-ph].

[4] J. R. Batley et al. [NA48/2 Collaboration], Phys. Lett. B 746, 178 (2015) [arXiv:1504.00607 [hep-ex]].

[5] E. M. Riordan et al., Phys. Rev. Lett. 59, 755 (1987).

[6] P. H. Gu and X. G. He, arXiv:1606.05171 [hep-ph].

[7] J. L. Feng, B. Fornal, I. Galon, S. Gardner, J. Smolinsky, T. M. P. Tait and P. Tanedo, arXiv:1608.03591 [hep-ph].

[8] M. Deniz et al. [TEXONO Collaboration], Phys. Rev. D 81, 072001 (2010) [arXiv:0911.1597 [hep-ex]].

[9] C. S. Chen, G. L. Lin, Y. H. Lin and F. Xu, arXiv:1609.07198 [hep-ph].

[10] G. Angloher et al. [CRESST Collaboration], arXiv:1509.01515 [astro-ph.CO].

[11] J. Barreto et al. [DAMIC Collaboration], Phys. Lett. B 711, 264 (2012) [arXiv:1105.5191 [astro-ph.IM]].

[12] R. Agnese et al. [SuperCDMS Collaboration], [arXiv:1509.02448 [astro-ph.CO]].

[13] D. S. Akerib et al. [LUX Collaboration], Phys. Rev. Lett. 116, 161301 (2016) [arXiv:1512.03506 [astro-ph.CO]].

[14] R. Essig, A. Manalaysay, J. Mardon, P. Sorensen and T. Volansky, Phys. Rev. Lett. 109, 021301 (2012) [arXiv:1206.2644 [astro-ph.CO]].

[15] R. Essig, M. Fernandez-Serra, J. Mardon, A. Soto, T. Volansky and T. T. Yu, JHEP 1605, 046 (2016) [arXiv:1509.01598 [hep-ph]]. 